\title{
Ludwig Tieck's Puss-in-Boots and Theater of the Absurd: A Commentated bilingual edition
}

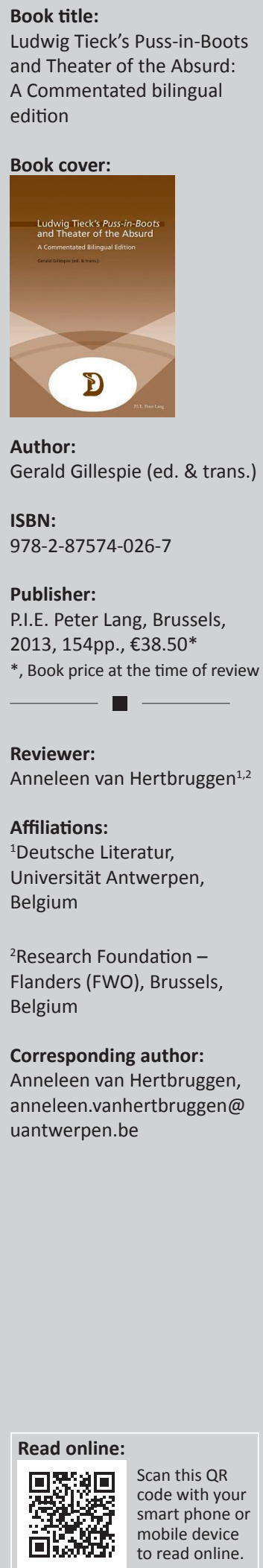

Author:

Gerald Gillespie (ed. \& trans.)

ISBN:

978-2-87574-026-7

Publisher:

P.I.E. Peter Lang, Brussels,

2013, 154pp., €38.50*

*, Book price at the time of review

$\square$

Reviewer:

Anneleen van Hertbruggen ${ }^{1,2}$

\section{Affiliations:}

${ }^{1}$ Deutsche Literatur,

Universität Antwerpen,

Belgium

${ }^{2}$ Research Foundation Flanders (FWO), Brussels, Belgium

Corresponding author: Anneleen van Hertbruggen, anneleen.vanhertbruggen@ uantwerpen.be

Read online:

Scan this QR code with your smart phone or mobile device to read online.

Gerald Gillespie übersetzte schon Luces de Bohemia, ein absurdes iberisches Werk von Ramón María del Valle-Inclán und in Zusammenarbeit mit Anthony Zahareas auch Ernst Klingemanns Die Nachtwachen von Bonaventura. Wegen seines Interesses am absurden Theater übersetzte er Der gestiefelte Kater in den 1970er Jahren zum ersten Mal ins Englische. Heute bietet Gillespie mit seinem Buch Ludwig Tieck's Puss-in-Boots and Theater of the Absurd. A commentated bilingual Edition eine zweisprachige und kommentierte Neuausgabe dieses Stückes an. Die Zielsetzung des vorliegenden Buches wird wahrscheinlich am besten mit einem Satz aus dem Vorwort des Herausgebers zusammengefasst:

Since Puss-in-Boots in English has meanwhile gone out of print, I have decided to bring it back in a commentated form as a helpful - and eminently playable - exemplar of Romantic imagination and one well-suited to illustrate the theatrical revolution ancestral to several strands of writing at the turn of the nineteenth to the twentieth century, in addition to Tieck's general precursor role in an earlier fin-de-siècle. (Gillespie 2013:10)

Gillespie macht diese Komödie, die das französische Kindermärchen Le maître Chat ou le chat botté aus der Sammlung Histoires ou Contes du temps passé - Contes de ma mère l'Oye (1697) von Charles Perrault als Vorlage hat, dem modernen englischsprachigen Leser nicht nur mittels der Übersetzung, sondern auch wegen der ausführlichen Einführung in absurdes Theater und Ludwig Tieck als Autor der Romantik zugänglich.

Das Buch umfasst zwei große Teile: Zunächst eine Einführung, in der der romantische Hintergrund des Stückes und Tiecks Einfluss auf das deutsche Drama geschildert werden, und dann der zweisprachige und kommentierte Theatertext. Die Einführung besteht aus zwei Kapiteln. Im ersten Kapitel der Einführung wird Ludwig Tieck im Kontext der Romantik geschildert. Obwohl er heutzutage als eine der Schlüsselfiguren der Gestaltung romantischer Genres und Muster gilt, schildert Gillespie ausführlich, dass Tieck die Kernjahrzehnte der romantischen Epoche weitaus überlebte. Er beschreibt die Kontinuität und spirituelle Ähnlichkeiten zwischen dem romantischen Drama und neuen Richtungen, die während des Fin de Siècle und zu Beginn des 20. Jahrhunderts entstanden sind. Obwohl Tieck zeitlich gesehen der Frühromantik angehört, spielt er in seinen Werken mit den Begrenzungen der verschiedenen Stilrichtungen. Man sollte ihn denn auch, so Gillespie, in einem größeren Kontext, in dem symbolistische, absurde und existentielle Charakteristiken eingeschlossen werden, betrachten (S. 14). Außerdem überschreite er oft die Grenzen der literarischen Gattungen:

Tieck's crossing of generic boundaries between prose fiction, drama, and cultural commentary strikingly exhibits the new Romantic readiness around 1800 to subordinate the structures of literature to the poet's creative mind. (S. 15)

Obwohl Tieck schon im ersten Teil dieses Kapitels als ein ,multifaceted and prolific writer' (S. 15) beschrieben wird, wird dies erst im zweiten Teil, in dem Gillespie eine kurze Biographie Tiecks darstellt, plausibel gemacht. Seine Bekanntschaft mit großen Namen wie Novalis, Fichte, den Brüdern Schlegel, Goethe und Schiller werden aufgezeichnet. Außerdem wird klar, dass Tieck auch beruflich eine sehr vielseitige Person war. Gillespie erwähnt Tiecks Tätigkeit als Theaterkritiker, Essayist, Übersetzer, Herausgeber und Autor (S. 22-23). Gillespie beschreibt Tiecks ,Dresdner Periode', in der er zuerst als Theaterkritiker für die Dresdner Abendzeitung und später als Dramaturg am Hoftheater und Herausgeber seiner eigenen Werke und der Werke von Kleist, Maler, Müller, Lenz und so weiter, arbeitete, als bezeichnend für seine Evolution von Romantiker zu Realist (S. 27). Vor allem in Tiecks Bewunderung für das Frühwerk von Kleist und wegen Tiecks Herausgabe von Kleists Stücke Der Prinz von Homburg und Die Hermannsschlacht ist

How to cite this book review: Van Hertbruggen, A., 2016, 'Ludwig Tieck's Puss-in-Boots and Theater of the Absurd: A Commentated bilingual edition (Book Review)', Literator 37(1), a1134. http://dx.doi.org/10.4102/lit.v37i1.1134

Copyright: @ 2016. The Authors. Licensee: AOSIS. This work is licensed under the Creative Commons Attribution License. 
laut Gillespie ein Zeichen von Tiecks ,drift toward realism (S. 27) zu sehen. Das zweite Kapitel der Einführung trägt als


der Suche nach einem Theater]. Gillespie versucht die Evolution des absurden Theaters zu erklären, indem er das Stück Der gestiefelte Kater (Tieck 1797) zentral stellt. Viele haben versucht, Tiecks Beispiel $\mathrm{zu}$ folgen, aber nur Eichendorff, Schnitzler und Hofmannsthal haben es, so Gillespie, geschafft, die Charakteristiken der Tieckschen Komödie, wie zumBeispiel, den Gebrauch italienischer Masken, den Auftritt mythischer Figuren, das Motiv eines Spiels in einem Spiel und so weiter, in ihren eigen Werken erfolgreich $\mathrm{zu}$ verarbeiten. Aber erst Pirandello habe die Phantasie einen richtigen Platz im Drama geben können (S. 29). Gillespie beschreibt Tieck als den geistigen Vorläufer des Dramas von Pirandello zu Dürrenmatt und Ionesco. Diese Art von Phantasie hat also ein Jahrhundert für ihre Suche nach einem Theater gebraucht (S. 30). Auch später noch hat Tiecks Einfluss auf neue Theaterstücke nachgewirkt. So erwähnt Gillespie Tankred Dorsts (1963) Adaptation Der gestiefelte Kater oder wie man das Spiel spielt, im Jahre 1963 uraufgeführt (S.31) und auch die Novelle The Master and Margarita von Mikhail Afanasievich Bulgakov (1967), die 1966-1967 posthum herausgegeben wurde, weist strukturelle Analogien zu Tiecks Der gestiefelte Kater auf (S.32). Anhand eines knappen Vergleichs mit unter anderen E.T.A. Hoffmann (S. 35) und Bertolt Brecht (s. 34) schildert Gillespie einen guten Einblick in einige Charakteristiken des absurden Theaters, zum Beispiel, die Zerstörung der Illusion, das Aus-der-Rolle-Fallen der Schauspieler und das ,Spiel im Spiel' (S. 34). Im letzten Teil dieses Kapitels bereitet Gillespie den Leser noch ganz spezifisch auf das Stück Der gestiefelte Kater vor. Er behandelt sowohl strukturelle als auch inhaltliche Aspekte des Dramas und weist den Leser schon auf Charakteristiken des Absurden Theaters in den unterschiedlichen Akten und Szenen.

Der zweite Teil des Buches umfasst die bilinguale Fassung des Stückes: Auf der linken Seite die deutsche Version, auf der rechten Seite ihre englische Übersetzung. Gillespie bietet dem Leser eine sehr textnahe englische Übersetzung. Außerdem erklärt er anhand der Endnoten auch Eigenheiten der deutschen Sprache im Originaltext, zum Beispiel, Gebrauch der Höflichkeitsform (S.65, Fn. 15), Dialektwörter (S.51, Fn. 3), Wortspiele (S.145, Fn. 45). Insgesamt fügt er dem Theatertext 52 Endnoten hinzu. Nicht alle Endnoten umfassen sprachliche Erläuterungen, Gillespie macht den
Leser auch auf Referenzen auf andere Stücke aufmerksam. So ist auch dem Leser gedient, der sich nicht unbedingt mit romantischer Literatur auskennt. Referenzen auf Don Carlos von Schiller, Die neuen Arkadier von Christian August Vulpius, Die Zauberflöte von Mozart, die Komödien Die Geschwister vom Lande und Die Entführung von Johann Friedrich Jünger, Hesperus von Jean Paul und viele andere werden auf diese Weise nicht vom Leser übersehen.

Diese kommentierte Ausgabe bietet sich als ein hilfreiches Exemplar romantischer Phantasie an. Der Theatertext in Kombination mit den erläuternden Endnoten und der ausführlichen Einführung in das Absurde Theater und die Einflüsse auf Ludwig Tieck haben zur Folge, dass der über 200 Jahre alte Text von Ludwig Tieck auch für heutige, englischsprachige LeserInnen, sehr aufschlussreich ist. Sowohl der deutsche Originaltext als auch die englische Übersetzung lassen sich sehr flüssig lesen und die extra dargebotenen Informationen tragen wesentlich zum ganzen Textverständnis bei. Kritisch soll aber die Vielfalt an Typfehlern angemerkt werden, sowohl im englischen (15 Shakspeares; 129 hack; 145 modem English) als auch im deutschen Text (16 Dickhtkunst; 31 gestielefte; 54 Publilum; 88 Augenhlick). Wenn man aber über diese Schönheitsfehler hinweg sehen kann, kommt es einem beim Lesen so vor, als komme Der gestiefelte Kater aus dem Buch wieder zum Leben.

\section{Literaturverzeichnis}

Bulgakov, M.A., 1967, The Master and Margarita, übersetzt von M. Glenny, Harper \& Row, New York.

Del Valle-Inclán, R.M., 1976, Luces de Bohemia/Bohemian Lights, übersetzt von G. Gillespie \& A.N. Zahareas, Edinburgh University Press, Edinburgh.

Dorst, T., 1963, Ludwig Tieck/Tankred Dorst: Der gestiefelte Kater oder wie man das Spiel spielt, Kiepenheuer \& Witsch, Köln. (Collection Theater, 15).

Gillespie, G. (ed. \& trans.), 2013, Ludwig Tieck's Puss-in-Boots and Theater of the Absurd. A commentated bilingual edition, P.I.E. Peter Lang, Brussel.

Jünger, J.F., 1791, Die Entführung.

Jünger, J.F., 1792, Die Geschwister vom Lande.

Klingemann, E.A.F., 1972, Die Nachtwachen des Bonaventura: The Nightwatches of Bonaventura, übersetzt von G. Gillespie, Edinburgh University Press, Edinburgh.

Mozart, W.A., 1794, Die Zauberflöte.

Paul, J., 1795, Hesperus.

Perrault, C., 1697, Le maître Chat ou le chat botté, in Histoires ou Contes du temps passé - Conte de ma mère l'Oye.

Tieck, L., 1797, 'Der gestiefelte Kater', in Volksmärchen herausgegeben von Peter Lebrecht, 2. Bd., Carl August Nicolai, Berlin.

Tieck, L., 1972, Der gestiefelte Kater: Puss-in-Boots, übersetzt von G. Gillespie, Edinburgh University Press, Edingburgh.

Von Schiller, F., 1787, Don Carlos.

Vulpius, C.A., 1796, Die neuen Arkadier. 